\title{
Adsorption of naphthalene on zeolite from aqueous solution
}

\author{
Chiung-Fen Chang ${ }^{\text {a }}$, Ching-Yuan Chang ${ }^{\mathrm{a}, *}$, Ken-Hung Chen ${ }^{\mathrm{a}}$, Wen-Tien Tsai ${ }^{\mathrm{b}}$, Je-Lueng Shie ${ }^{\mathrm{a}}$, \\ Yi-Hung Chen ${ }^{\text {a }}$ \\ ${ }^{a}$ Graduate Institute of Environmental Engineering, National Taiwan University, Taipei 106, Taiwan \\ ${ }^{\mathrm{b}}$ Department of Environmental Engineering and Health, Chia Nan University of Pharmacy and Science, Tainan 717, Taiwan
}

Received 18 January 2004; accepted 14 April 2004

Available online 18 May 2004

\begin{abstract}
Polynuclear aromatic hydrocarbons (PAHs), which are environmental hormones and carcinogens, are viewed as the priority pollutants to deal with by many countries. Most PAHs are hydrophobic with high boiling and melting points and high electrochemical stability, but with low water solubility. Compared with other PAH species, naphthalene has less toxicity and is easily found in the environment. Thus, naphthalene is usually adopted as a model compound to examine the environmental and health aspects of PAHs. This study attempted to use an adsorption process to remove naphthalene from a water environment. The adsorption equilibrium of naphthalene on zeolite from water-butanol solution, which is a surfactant-enriched scrubbing liquid, was successfully evaluated by Langmuir, Freundlich, and linear isotherms. Among the tested kinetics models in this study (e.g., pseudo-first-order, pseudo-second-order, and Elovich rate equations), the pseudo-second-order equation successfully predicted the adsorption.
\end{abstract}

(c) 2004 Elsevier Inc. All rights reserved.

Keywords: Naphthalene; Zeolite; Adsorption isotherms; Adsorption kinetics; Pseudo-first-order process; Pseudo-second-order process; Elovich rate equation

\section{Introduction}

Polynuclear aromatic hydrocarbons (PAHs), which many countries view as the priority pollutants to deal with, are environmental hormones and carcinogens. PAHs are made up of only carbon and hydrogen, and can be divided into two groups: kata-annellated and peri-condensed [1]. The saturated vapor pressures of PAHs at $298 \mathrm{~K}$ are lower than $0.1 \mathrm{~mm} \mathrm{Hg}$ and belong to semivolatile organic compounds (SVOCs). Most PAHs are hydrophobic with high boiling and melting points and possess low water solubility and electrochemical stability. Therefore, they can exist and be accumulated in the environment for long times. The sources of PAHs can be divided into two categories, artificial and natural sources, in which the amount of the former is far greater than that of the later and surpasses the self-purification capacity.

Due to the refractoriness and difficulty of biological degradation of PAHs, it is found that PAHs have accumulated in the air, water bodies, soil, and food. Therefore,

\footnotetext{
* Corresponding author. Fax: +886-2-2363-8994.

E-mail address: cychang3@ntu.edu.tw (C.-Y. Chang).
}

immediate attention to the effective treatment of PAHs is needed. The methods for treating PAHs mainly include biodegradation [2,3], scrubber absorption [4-6], high-energy electron beam irradiation [7], ozonation, and catalytic combustion. In order to avoid a secondary public nuisance, it is suitable to treat PAHs with medium-high concentrations by catalytic combustion and ozonation. The adsorption process is widely applied to organic compound removal in water/wastewater treatment with high removal efficiency. Although activated carbon is the most commonly used sorbent, it still has some limitations in application, such as flammability, difficulty of regenerating adsorbed high-boiling-point organics, etc. In contrast to the activated carbon, zeolite (aluminosilicates renowned for their microporosity, catalytic properties, and extensive applications) can be used in more specific situations with the advantages of the framework's open structure, rich ion-exchange chemistry, etc. [8], but without the disadvantages of activated carbon. Therefore, zeolites were used as adsorbents to concentrate organics from streams [9-14].

Naphthalene is a natural constituent of coal tar and commonly used as a wood preservative, moth repellent, and raw material to produce methylanthranilate, phthalate es- 
ters, chloronaphthalene, synthetic resins, etc. The sources of naphthalene in the air are mainly from the burning of coal and oil, the use of mothballs, the production of coal tar, etc. Being compared with other PAHs species, naphthalene, the simplest PAH, has less toxicity and is easily found in the environment. Therefore, the adoption of naphthalene as the target compound in this study can not only provide useful information for the treatment of naphthalene but also be viewed as the primary method of inquiry for dealing with complicated PAHs. The aim of this study is to investigate the feasibility of using hydrophobic zeolites to remove naphthalene from a water-butanol solution which serves as a surfactant-enriched scrubbing liquid. The adsorption of naphthalene on zeolites from water-butanol solutions is further evaluated by the common isotherms (e.g., Langmuir, Freundlich, and linear isotherms) and kinetic models (e.g., pseudo-first-order, pseudo-second-order, and Elovich rate equations).

\section{Materials and methods}

\subsection{Adsorbent}

Hydrophobic zeolite (type: DAY-Zeolith, Degussa, Germany) with a particle size range between 20 and 30 meshes (with sieve opening of 0.841 and $0.595 \mathrm{~mm}$ ) was used as the adsorbent. The mean particle size $d_{\mathrm{p}}$ of DAY zeolite is $0.72 \mathrm{~mm}$. The physical characteristics of DAY zeolite are shown in Table 1. The pretreatment of the adsorbent comprised several steps. First, the adsorbent was washed with distilled water to remove the crushed fines. Then, it was dried overnight at $383 \mathrm{~K}$ in a vacuum oven and then stored in a desiccator. Finally, it was wetted in the water-butanol solution under vacuum, prior to the adsorption experiments. The weights of the adsorbents used in this study ranged between 0.5 to $3 \mathrm{~g}$.

Table 1

Physical characteristics of hydrophobic DAY zeolite

\begin{tabular}{ll}
\hline Property & Value \\
\hline Mesh size & $20-30$ \\
Average particle diameter, ${ }^{\mathrm{a}} d_{\mathrm{p}}(\mathrm{mm})$ & 0.72 \\
Specific external surface area, ${ }^{\mathrm{b}} a_{\mathrm{S}}\left(\mathrm{m}^{2} / \mathrm{kg}\right)$ & 5.48 \\
Average true particle density, ${ }^{\mathrm{a}} \rho_{\mathrm{s}}\left(\mathrm{kg} / \mathrm{m}^{3}\right)$ & 2380 \\
Apparent particle density, ${ }^{\mathrm{a}} \rho_{\mathrm{p}}\left(\mathrm{kg} / \mathrm{m}^{3}\right)$ & 1520 \\
Particle porosity, ${ }^{\mathrm{c}} \varepsilon_{\mathrm{p}}(-)$ & 0.36 \\
BET specific surface area $^{\mathrm{a}}\left(\mathrm{m}^{2} / \mathrm{g}\right)$ & 601 \\
Total pore volume ${ }^{\mathrm{a}}\left(\mathrm{cm}^{3} / \mathrm{g}\right)$ & 0.237 \\
Average pore diameter $(\AA)$ & 15.8 \\
Average pore hydraulic radius ${ }^{\mathrm{d}}(\AA)$ & 3.01 \\
\hline${ }^{\mathrm{a}}$ Data source: $\operatorname{Ref}^{\mathrm{a}}[14]$. & \\
$\mathrm{b}$ Assumed as a spherical particle and calculated using $a_{\mathrm{s}}=6 /\left(\rho_{\mathrm{p}} d_{\mathrm{p}}\right)$. \\
${ }^{\mathrm{c}}$ Calculated using $\varepsilon_{\mathrm{p}}=1-\left(\rho_{\mathrm{p}} / \rho_{\mathrm{s}}\right)$. & \\
d Data from the analysis by the micropore method (MP method) with the \\
pore size between 2.4 and $17.2 \AA$.
\end{tabular}

\subsection{Adsorbate}

Naphthalene $\left(\mathrm{C}_{10} \mathrm{H}_{8}\right)$, with a molecular weight of 128.16 , was scintillation grade, provided by Merck, and was used as the representative compound of PAHs in this study. The initial concentrations of naphthalene for adsorption experiments were 4.5 to $33 \mathrm{~g} \mathrm{~m}^{-3}$. A certain amount of naphthalene was dissolved in 1-butanol (reagent grade supplied by Nacalai Tesque, Inc., Japan) of $37 \mathrm{ml}$, and subsequently diluted by distilled water of $3668 \mathrm{ml}$ to prepare solutions at various concentrations, the total volume of which is fixed at $3705 \mathrm{ml}$.

\subsection{Aqueous systems}

Due to the low water solubility of naphthalene, the waterbutanol solution was used as the aqueous solution. It is noted that butanol is a good solvent used to enhance the adsorption of hydrophobic naphthalene from the hydrophobic environment, forming the naphthalene-containing solutions. The water-butanol solution was composed of $37 \mathrm{ml}$ butyl alcohol and $3668 \mathrm{ml}$ distilled water.

\subsection{Analytical measurements}

Prior to the analysis, all the samples were filtrated through a $0.45-\mu \mathrm{m}$ membrane. Two analyzers, i.e., a total organic carbon (TOC) analyzer (O.I.C. M-700/carbon analyzer Dohrmann DC-80), and UV spectrophotometer (GBC UV-visible Cintra 20 spectrometer) were used to determine the concentrations of butanol and naphthalene, respectively, in this study. The wavelength used in the UV spectrophotometer for naphthalene was $276.3 \mathrm{~nm}$.

\subsection{Adsorption behavior}

Samples with various weight ratios of naphthalene to zeolite were prepared to get various values of final equilibrium concentrations. The experiments were performed at $298 \mathrm{~K}$ in the completely stirred tank reactor (CSTR) until the concentrations of filtrate did not change within a range of $\pm 3 \%$. Since it is preferable to use weight concentration units for investigating the removal efficiency in wastewater treatments, the units of $\mathrm{g} \mathrm{m}^{-3}$ and $\mathrm{g} \mathrm{kg}^{-1}$ for the liquid and solid phases were used for the organics in this study. The amount of naphthalene adsorbed at time $t$ and equilibrium were calculated using the equations

$q_{t}=\left(C_{0}-C_{t}\right) \times V / W$,

$q_{\mathrm{e}}=\left(C_{0}-C_{\mathrm{e}}\right) \times V / W$,

where $q_{\mathrm{e}}$ and $q_{t}$ are the adsorbate concentrations in the solid at equilibrium and time $t$, respectively; $C_{0}, C_{t}$, and $C_{\mathrm{e}}$ are the initial concentration, the liquid-phase concentration at time $t$, and the equilibrium concentration of naphthalene, respectively; $V$ is the volume of the aqueous solution and equal to $3.705 \mathrm{~L}$; and $W$ is the mass of zeolite used in the experiments. 


\section{Results and discussion}

\subsection{Adsorption equilibria}

In order to clarify the existence of competitive adsorption between naphthalene and butanol on zeolite, the adsorption of butanol on DAY zeolite was first conducted, as illustrated in Fig. 1. The result shows that butanol was not adsorbed onto the zeolite, since it did not possess competitive adsorption on zeolite from the water-butanol solution containing naphthalene. For investigation of the adsorption of naphthalene on DAY zeolite in this study, the empirical Freundlich and Langmuir isotherms, which correspond to the heterogeneous and homogeneous adsorbent surfaces, respectively, were used to correlate the experimental data as follows:

$q_{\mathrm{e}}=k_{\mathrm{F}} C_{\mathrm{e}}^{1 / n_{\mathrm{F}}}$,

$q_{\mathrm{e}}=\frac{Q_{\mathrm{L}} K_{\mathrm{L}} C_{\mathrm{e}}}{1+K_{\mathrm{L}} C_{\mathrm{e}}}$

$k_{\mathrm{F}}$ and $n_{\mathrm{F}}$ are the Freundlich isotherm constants. $Q_{\mathrm{L}}$ and $K_{\mathrm{L}}$ are the Langmuir isotherm constants, representing the monolayer adsorption capacity and equilibrium constant, respectively. The constants in the models can be obtained by linearizing the above equations as follows:

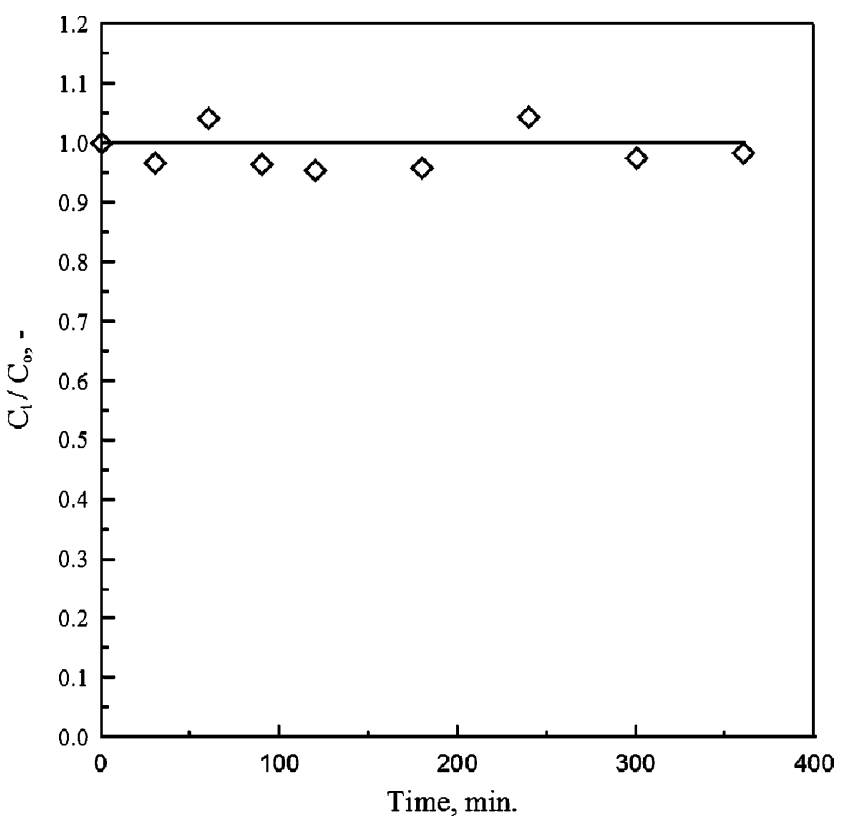

Fig. 1. Dependence of butanol concentration $\left(C_{t}\right)$ on time using DAY zeolite as the adsorbent in a stirred tank at $800 \mathrm{rpm}$. The volume of solution is $3.705 \mathrm{~L}$. The mass of zeolite is $3 \mathrm{~g}$. The initial concentration of butanol $\left(C_{0}\right)$ is $11 \mathrm{~g} / \mathrm{m}^{3}$.

$$
\begin{aligned}
& \ln q_{\mathrm{e}}=\ln k_{\mathrm{F}}+n_{\mathrm{F}} \ln C_{\mathrm{e}}, \\
& \frac{1}{q_{\mathrm{e}}}=\frac{1}{Q_{\mathrm{L}} K_{\mathrm{L}}} \frac{1}{C_{\mathrm{e}}}+\frac{1}{Q_{\mathrm{L}}} .
\end{aligned}
$$

The results are shown in Table 2 and Fig. 2. Both adsorption isotherms can well predict the adsorption of naphthalene on zeolite with high correlation coefficients $\left(r^{2}\right)$. It is noted that the value of $n_{\mathrm{F}}$ is very close to 1 , suggesting that the adsorption of naphthalene on zeolite approximately follows a linear isotherm. Furthermore, according to the lower limit of the Langmuir isotherm (i.e., $K_{\mathrm{L}} C_{\mathrm{e}} \ll 1$ ), the Langmuir isotherm can be reduced to a linear isotherm as follows:

$q_{\mathrm{e}}=K_{\mathrm{d}} C_{\mathrm{e}}$,

where $K_{\mathrm{d}}$ is the equilibrium distribution coefficient describing the distribution of liquid in the adsorbent. Equation (7) is similar to Henry's law $\left(q_{\mathrm{e}}=K_{\mathrm{H}} C_{\mathrm{e}}\right)$, of which the constant $K_{\mathrm{H}}$ describes the gas solubility in the liquid. Compared with the Freundlich and Langmuir isotherms, the linear isotherm was also tested to predict the experimental data, as seen in Table 2 and Fig. 2. The results showed that good applicability was also obtained by the linear isotherm. As a result, Freundlich, Langmuir, and linear isotherms were all suitable to be applied in predicting the adsorption of naphthalene on DAY zeolite from water-butanol solution.

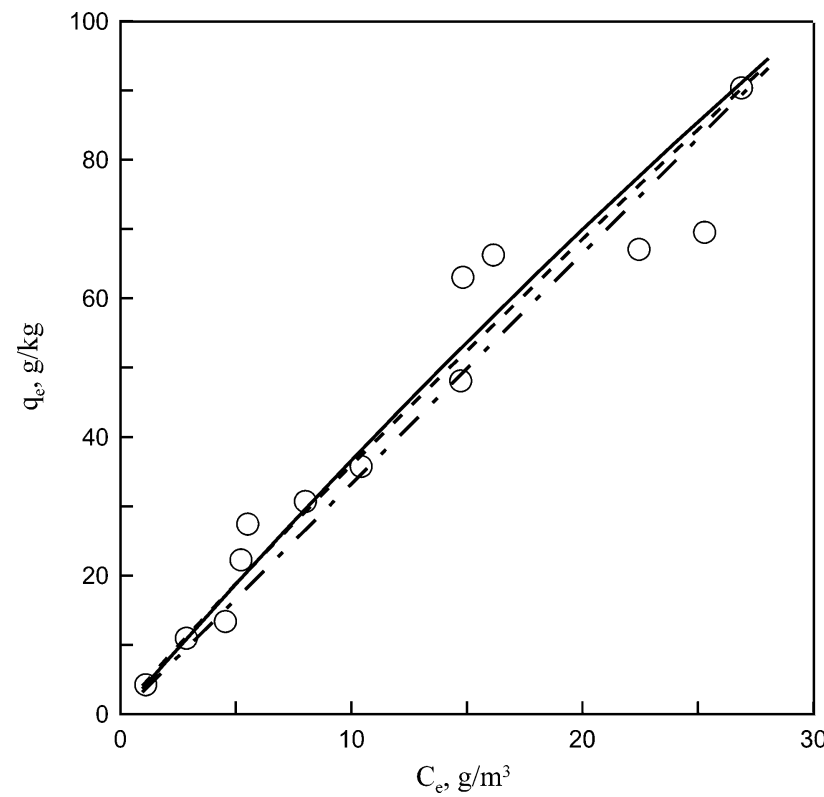

Fig. 2. Isothermal adsorption of naphthalene on DAY zeolite by dynamic experiments in the stirred tank at $800 \mathrm{rpm} .(\mathrm{O}),(-),(--)$ and (--): experimental data, simulated by Langmuir, Freundlich, and linear isotherms.

Table 2

\begin{tabular}{|c|c|c|c|c|c|c|c|}
\hline \multicolumn{3}{|l|}{ Freundlich isotherm } & \multicolumn{3}{|c|}{ Langmuir isotherm } & \multicolumn{2}{|c|}{ Linear isotherm } \\
\hline$k_{\mathrm{F}}(\mathrm{g} / \mathrm{kg}) /\left(\left(\mathrm{g} / \mathrm{m}^{3}\right)^{1 / n_{\mathrm{F}}}\right)$ & $n_{\mathrm{F}}(-)$ & $r^{2}$ & $K_{\mathrm{L}}\left(\mathrm{m}^{3} / \mathrm{kg}\right)$ & $Q_{\mathrm{L}}(\mathrm{g} / \mathrm{kg})$ & $r^{2}$ & $K_{\mathrm{d}}\left(\mathrm{m}^{3} / \mathrm{kg}\right)$ & $r^{2}$ \\
\hline 4.215 & 1.074 & 0.9683 & 0.005 & 769.231 & 0.9894 & 3.324 & 0.9149 \\
\hline
\end{tabular}

Isotherm parameters for adsorption of naphthalene onto DAY zeolite in the water-butanol solution 


\subsection{Adsorption kinetics}

Numerous publications in literature on adsorption kinetics are available [14-18]. The mass transfers occur within the boundary layer around the adsorbent and proceed in the liquid-filled pores or along the walls of the pores of adsorbent, which are called external and internal mass transfers, respectively. The typical kinetic models normally consider both the external and internal mass transfers. Examples of these models are film-pore diffusion, film-surface diffusion, pore diffusion, surface diffusion, and combined pore and surface diffusion models. The models involve a complicated mathematical computation to obtain the related diffusion coefficients of the models. Furthermore, the mass transfers of adsorption often involve many controlling mechanisms, of which the individual contribution may not be clearly recognized, at the same time during the course to approach adsorption equilibria. Therefore, for the simplicity and practical use of engineering applications, the global kinetic expressions such as Lagergren pseudo-first-order, pseudo-second-order, and Elovich rate equations, were adopted to describe the adsorption kinetics in the study [18] by means of the lumped analysis of kinetics data.

\subsubsection{Pseudo-first-order process}

For the pseudo-first-order process, the Lagergren equation was expressed as

$\frac{d q_{t}}{d t}=k_{\mathrm{e} 1}\left(q_{\mathrm{e}}-q_{t}\right)$.

Integrating Eq. (8) with the conditions $\left(q_{t}=0\right.$ at $t=0 ; q_{t}=$ $q_{t}$ at $t=t$ ) gives

$\ln \left(q_{\mathrm{e}}-q_{t}\right)=\ln \left(q_{\mathrm{e}}\right)-k_{\mathrm{e} 1} t$, where $q_{t}$ and $q_{\mathrm{e}}$ have the same meaning as described earlier and $k_{\mathrm{e} 1}$ is the equilibrium rate constant of pseudo-first-order sorption.

\subsubsection{Pseudo-second-order process}

The pseudo-second-order process can be presented as follows:

$\frac{d q_{t}}{d t}=k_{\mathrm{e} 2}\left(q_{\mathrm{e}}-q_{t}\right)^{2}$.

Integrating Eq. (10) with the conditions $\left(q_{t}=0\right.$ at $t=0$; $q_{t}=q_{t}$ at $t=t$ ) yields

$\frac{t}{q_{t}}=\frac{1}{k_{\mathrm{e} 2} q_{\mathrm{e}}^{2}}+\frac{t}{q_{\mathrm{e}}}$,

where $k_{\mathrm{e} 2}$ is the equilibrium rate constant of the pseudosecond-order sorption.

\subsubsection{Elovich rate equation}

The Elovich equation is as follows:

$\frac{d q_{t}}{d t}=a \exp \left(-b q_{t}\right)$.

Integrating Eq. (12) with the conditions $\left(q_{t}=0\right.$ at $t=0$; $q_{t}=q_{t}$ at $\left.t=t\right)$ and subsequently linearizing the integrated equation result in

$q_{t}=\frac{1}{b} \ln (a b)+\frac{1}{b} \ln \left(t+t_{0}\right)$,

where $a$ and $b$ are the parameters of the Elovich rate equation; $t_{0}$ is equal to $1 /(a b)$.

If $a b t \gg 1$, Eq. (13) can further be simplified as

$q_{t}=\frac{1}{b} \ln (a b)+\frac{1}{b} \ln (t)$.

Table 3

Parameters and determination coefficients $\left(R^{2}\right)$ of various kinetic models

\begin{tabular}{|c|c|c|c|c|c|c|c|c|c|c|}
\hline \multirow{2}{*}{$\begin{array}{l}\text { Initial concentration } C_{0} \\
\left(\mathrm{~g} / \mathrm{m}^{3}\right)\end{array}$} & \multicolumn{3}{|c|}{ Pseudo-first-order equation } & \multicolumn{3}{|c|}{ Pseudo-second-order equation } & \multicolumn{4}{|c|}{ Elovich rate equation } \\
\hline & $r^{2 \mathrm{a}}$ & $k_{\mathrm{e} 1}$ & $R^{2}$ & $r^{2 \mathrm{~b}}$ & $k_{\mathrm{e} 2}$ & $R^{2}$ & $r^{2 \mathrm{c}}$ & $a$ & $b$ & $R^{2}$ \\
\hline 5 & 0.9961 & 0.0244 & 0.8752 & 0.9950 & 0.00595 & 0.9874 & 0.9684 & 0.3048 & 0.9061 & 0.9684 \\
\hline 15 & 0.9924 & 0.0179 & 0.6030 & 0.9984 & 0.00227 & 0.9896 & 0.9540 & 1.1548 & 0.3402 & 0.9595 \\
\hline 23 & 0.9675 & 0.0109 & 0.9461 & 0.9990 & 0.00048 & 0.9959 & 0.9868 & 0.6838 & 0.1869 & 0.9895 \\
\hline 28 & 0.9975 & 0.0103 & 0.7467 & 0.9996 & 0.00045 & 0.9743 & 0.9639 & 1.3394 & 0.1723 & 0.9656 \\
\hline 33 & 0.9849 & 0.0089 & 0.9263 & 0.9950 & 0.00040 & 0.9792 & 0.9875 & 1.2813 & 0.1215 & 0.9875 \\
\hline
\end{tabular}

${ }^{a}$ Correlation coefficient for linear regression of Eq. (9).

b Correlation coefficient for linear regression of Eq. (11).

c Correlation coefficient for linear regression of Eq. (14).

Table 4

Comparison of equilibrium adsorption capacities $\left(q_{\mathrm{e}}\right)$

\begin{tabular}{llccc}
\hline $\begin{array}{l}\text { Initial concentration } C_{0} \\
\left(\mathrm{~g} / \mathrm{m}^{3}\right)\end{array}$ & $\begin{array}{l}\text { Experimental data } q_{\mathrm{e}} \\
(\mathrm{g} / \mathrm{kg})\end{array}$ & $\begin{array}{l}\text { Langmuir isotherm } q_{\mathrm{e}} \\
(\mathrm{g} / \mathrm{kg})\end{array}$ & $\begin{array}{l}\text { Pseudo-first-order equation } q_{\mathrm{e}} \\
(\mathrm{g} / \mathrm{kg})\end{array}$ & $\begin{array}{l}\text { Pseudo-second-order equation } q_{\mathrm{e}} \\
(\mathrm{g} / \mathrm{kg})\end{array}$ \\
\hline 5 & 4.22 & 4.21 & 3.87 & 4.96 \\
15 & 13.23 & 17.47 & 10.93 & 14.58 \\
23 & 22.00 & 20.23 & 21.78 & 25.64 \\
28 & 27.30 & 21.00 & 24.86 & 30.77 \\
33 & 31.23 & 29.59 & 29.18 & 37.88 \\
\hline
\end{tabular}




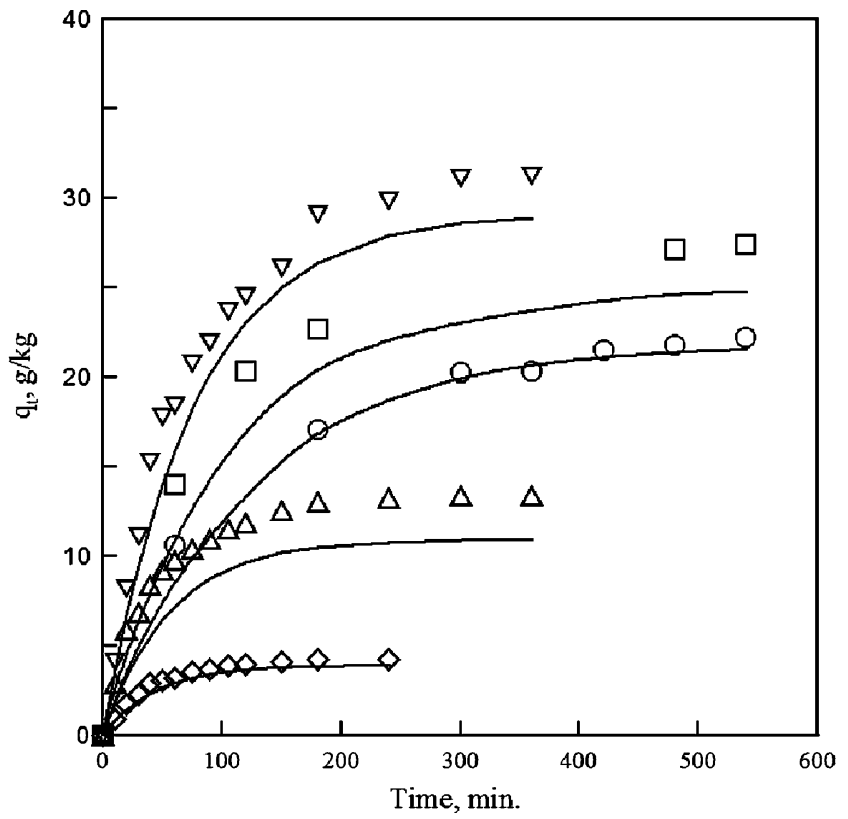

Fig. 3. Pseudo-first-order adsorption kinetics of naphthalene on zeolite at various values of $C_{0} \cdot(\diamond),(\triangle),(\bigcirc),(\square)$, and $(\nabla): C_{0}=5,15,23,28$, and $33 \mathrm{~g} / \mathrm{m}^{3}$, respectively. (-): simulated by pseudo-first-order kinetic equation.

The results are shown in Tables 3-4, and Figs. 3-5. In addition, the coefficient of determination $\left(R^{2}\right)$ used to compare the validities of the fitting of the three models is defined as

$R^{2}=1-\left[\sum\left(y_{\mathrm{e}}-y_{\mathrm{c}}\right)^{2} / \sum\left(y_{\mathrm{e}}-y_{\mathrm{m}}\right)^{2}\right]$,

where $y_{\mathrm{e}}, y_{\mathrm{c}}$, and $y_{\mathrm{m}}$ are the experimental and predicted data, and the average of the experimental values, respectively.

Comparing the values of $R^{2}$ of pseudo-first-order and second-order equations, the latter is better than the former and can be used to predict the adsorption kinetics of naphthalene on the zeolite. The values of $q_{\mathrm{e}}$ predicted by the pseudosecond-order equation are also in good agreement with the experimental data, as shown in Table 3. Among all the calculated $q_{\mathrm{e}}$ the data based on the pseudo-first-order equation are the closest to the experimental data. The Elovich rate equation is commonly used to describe the sorption behavior with a rapid equilibrium rate in the early period, while it slows down the equilibrium at later periods of the sorption process. The constants $a$ and $b$, in the Elovich rate equations represent the rate of sorption and surface coverage, respectively [16]. Along with increasing initial concentrations, the value of $b$ decreases due to the less available surface for naphthalene. Furthermore, the value of $a$ should increase with initial concentrations because of the higher driving force. However, this result was not obtained in this study, although the values of $R^{2}$ of prediction fitted by the Elovich rate equation are rather high. The physical meaning should overwhelm the determination coefficient. Therefore, the Elovich rate equation may not be suitable to describe the kinetics of naphthalene adsorbed onto the zeolite for this reason.

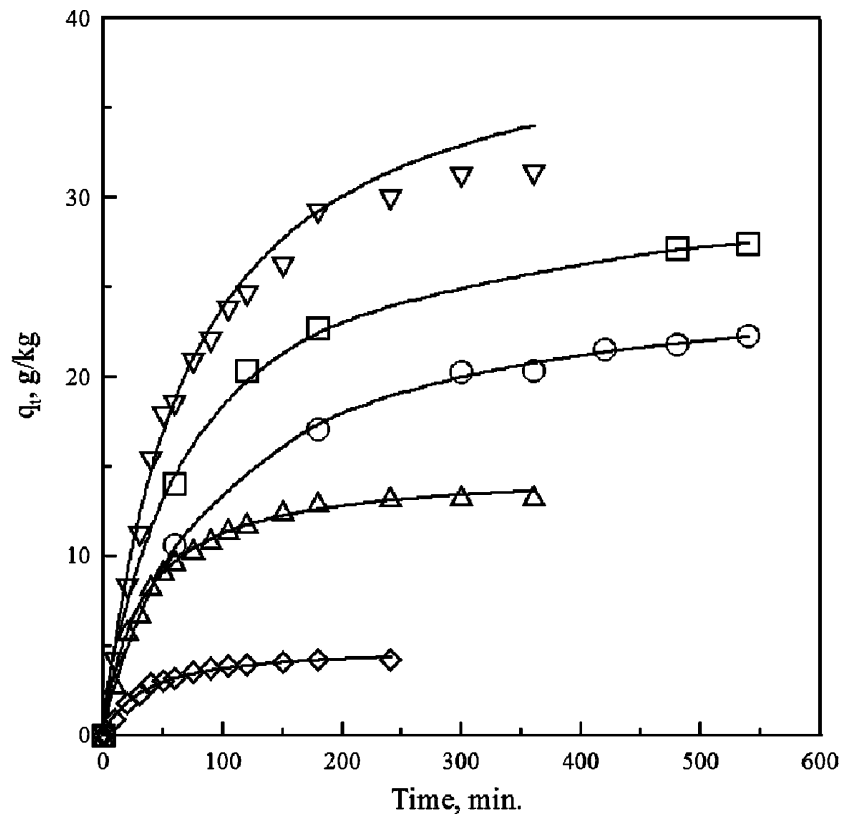

Fig. 4. Pseudo-second-order adsorption kinetics of naphthalene on zeolite at various values of $C_{0} .(\diamond),(\triangle),(\bigcirc),(\square)$, and $(\nabla)$ : $C_{0}=5,15,23,28$, and $33 \mathrm{~g} / \mathrm{m}^{3}$, respectively. (-): simulated by pseudo-second-order kinetic equation.

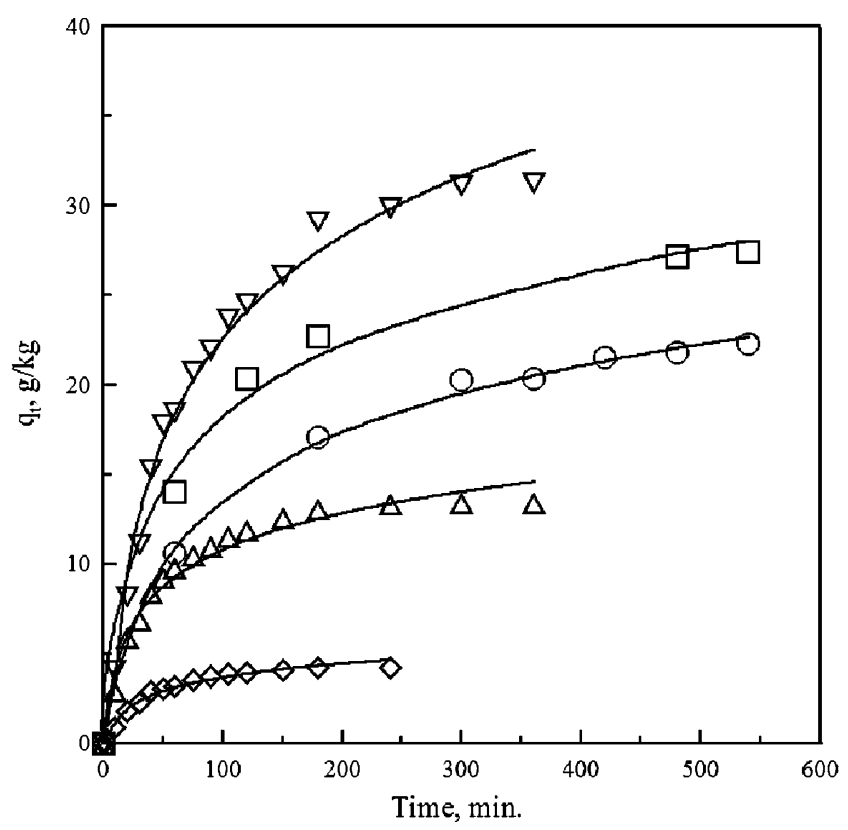

Fig. 5. Elovich adsorption kinetics of naphthalene on zeolite at various values of $C_{0} \cdot(\diamond),(\triangle),(\bigcirc),(\square)$, and $(\nabla): C_{0}=5,15,23,28$, and $33 \mathrm{~g} / \mathrm{m}^{3}$, respectively. (-): simulated by Elovich kinetic equation.

\section{Conclusions}

The adsorption behavior of naphthalene on the zeolite from water-butanol solution has been investigated in this study. Langmuir, Freundlich, and linear isotherms can be used to describe the adsorption equilibria of naphthalene on the zeolite. The simple kinetic model of the pseudo- 
second-order equation has been successfully applied to predict the adsorption of naphthalene on the zeolite. Although the pseudo-first-order equation can well predict the equilibrium capacity of naphthalene, the low coefficient of determination suggested that this model cannot be adapted to describe the kinetics. Regarding the Elovich rate equation, its value of parameter $a$, which represents the rate of adsorption, is inconsistent with the initial concentration. Therefore, it is not recommended to describe the adsorption kinetics in this study.

\section{Acknowledgment}

The authors thank the Powder Technology Laboratory of the Chemical Engineering Department of National Taiwan University for assistance in powder characterization.

\section{Appendix A. Nomenclature}

a Elovich rate equation constant representing rate of sorption, as specified in Eq. (12) $(\mathrm{g} / \mathrm{kg} / \mathrm{min})$

$a_{\mathrm{s}} \quad$ Specific external surface area $\left(\mathrm{m}^{2} / \mathrm{kg}\right)$

$b \quad$ Elovich rate equation constant representing surface coverage, as specified in Eq. (12) $(\mathrm{kg} / \mathrm{g})$

BET The specific surface area obtained following the Brunauer, Emmett, and Teller theory

$C_{\mathrm{e}} \quad$ Adsorbate concentration in the liquid phase at equilibrium with $q_{\mathrm{e}}\left(\mathrm{g} \mathrm{m}^{-3}\right)$

$C_{0} \quad$ Initial adsorbate concentration in the liquid phase $\left(\mathrm{g} \mathrm{m}^{-3}\right)$

$C_{t} \quad$ Adsorbate concentration in the liquid phase at time $t\left(\mathrm{~g} \mathrm{~m}^{-3}\right)$

$d_{\mathrm{p}} \quad$ Mean particle size $(\mathrm{mm})$

$k_{\mathrm{F}} \quad$ Freundlich isotherm constant as specified in Eq. (3), $(\mathrm{g} / \mathrm{kg}) /\left(\left(\mathrm{g} / \mathrm{m}^{3}\right)^{1 / n_{\mathrm{F}}}\right)$

$K_{\mathrm{d}} \quad$ Equilibrium distribution coefficient as specified in Eq. (7) $\left(\mathrm{m}^{3} / \mathrm{kg}\right)$

$K_{\mathrm{H}} \quad$ Equilibrium constant of Henry's law $\left(\mathrm{m}^{3} / \mathrm{mol}\right)$

$K_{\mathrm{L}} \quad$ Langmuir isotherm constant as specified in Eq. (4) $\left(\mathrm{m}^{3} / \mathrm{kg}\right)$

$k_{\mathrm{e} 1} \quad$ Equilibrium rate constant of pseudo-first-order equation as specified in Eq. (8) $(1 / \mathrm{min})$

$k_{\mathrm{e} 2} \quad$ Equilibrium rate constant of pseudo-second-order equation as specified in Eq. (10) $(\mathrm{kg} / \mathrm{g} / \mathrm{min})$

MP Micropore

$n_{\mathrm{F}} \quad$ Freundlich isotherm constant as specified in Eq. (3) $(-)$
PAHs Polynuclear aromatic hydrocarbons

$q_{\mathrm{e}} \quad$ Adsorbate concentration in solid phase at equilibrium with $C_{\mathrm{e}}(\mathrm{g} / \mathrm{kg})$

$q_{t} \quad$ Adsorbate concentration in solid phase at time $t$ $(\mathrm{g} / \mathrm{kg})$

$Q_{\mathrm{L}} \quad$ The monolayer adsorption capacity as specified in Eq. (4) $(\mathrm{g} / \mathrm{kg})$

$R^{2} \quad$ Determination coefficient defined by Eq. (15)

$r^{2} \quad$ Correlation coefficient

TOC Total organic carbon

$t \quad$ Adsorption time or elapsed time (min)

$t_{0} \quad 1 /(a b)(1 / \mathrm{min})$

$V \quad$ Volume of aqueous solution, equal to $3.705 \mathrm{dm}^{3}$

$W \quad$ Mass of zeolite used in experiments (g)

$y_{\mathrm{c}} \quad$ Experimental data as specified in Eq. (15)

$y_{\mathrm{e}} \quad$ Predicted values as specified in Eq. (15)

$y_{\mathrm{m}} \quad$ Average of the experimental values as specified in Eq. (15)

$\varepsilon_{\mathrm{p}} \quad$ Adsorbent porosity (-)

$\rho_{\mathrm{p}} \quad$ Apparent particle density $\left(\mathrm{kg} / \mathrm{m}^{3}\right)$

$\rho_{\mathrm{s}} \quad$ Average true particle density $\left(\mathrm{kg} / \mathrm{m}^{3}\right)$

\section{References}

[1] M. Zander, in: A. Bjrseth (Ed.), Handbook of Polycyclic Aromatic Hydrocarbons, Dekker, New York, 1983, pp. 1-26.

[2] T. Ebihara, P.L. Bishop, J. Environ. Eng. 128 (6) (2002) 505-513.

[3] H.N. Holma, K. Nieman, D.L. Sorensen, C.D. Miller, M.C. Martin, T. Borch, W.R. McKinny, R.C. Sims, Environ. Sci. Technol. 36 (6) (2002) 1276-1280.

[4] K. Friedman, Removal of Naphthalene from Coke Oven Gas, available at: http://www.lehigh.edu/ Kaf3/cchemdata/naphth.html, 1994.

[5] M. Platts, Thyssenkrupp Encoke USA, Qureshi, 2002.

[6] H. Huang, W. Lee, J. Environ. Eng. 128 (1) (2002) 60-67.

[7] W. Cooper, M.G. Nickelsen, R.V. Green, S.P. Mezyk, Radiation Phys. Chem. 65 (4-5) (2002) 571-577.

[8] S.L. Suib, Science 302 (5649) (2003) 1335-1336.

[9] D. Drohan, Metal Finishing 91 (11) (1993) 60-62.

[10] S.B. Riffat, Int. J. Energy Res. 18 (7) (1994) 657-664.

[11] N.A. Booker, E.L. Cooney, A.J. Priestly, Water Sci. Technol. 34 (9) (1996) 17-24.

[12] W.T. Tsai, C.Y. Chang, H.C. Lee, J. Environ. Sci. Health A 31 (1996) 995.

[13] I.V. Babich, A.D. Langeveld, W. Zhu, W.J.W. Bakker, J.A. Moulijn, Ind. Eng. Chem. Res. 40 (1) (2001) 257-363.

[14] C.Y. Chang, W.T. Tsai, C.H. Ing, C.F. Chang, J. Colloid Interface Sci. 260 (2003) 273-279.

[15] Y.S. Ho, G. McKay, Chem. Eng. J. 70 (1998) 115-124.

[16] H. Teng, C. Hsieh, Ind. Eng. Chem. Res. 38 (1999) 292-297.

[17] C.F. Chang, C.Y. Chang, W. Höll, Ind. Eng. Chem. Res. 42 (2003) 6904-6910.

[18] C.W. Cheung, J.F. Porter, G. McKay, Water Res. 35 (3) (2001) 605612. 